\title{
The response of clay plaster to temperature and RH sinusoidal variations
}

\author{
Valeria Cascione ${ }^{1, *}$, Denise Lim $^{1}$, Daniel Maskell ${ }^{1}$, Andy Shea ${ }^{1}$ and Pete Walker ${ }^{1}$ \\ ${ }^{1}$ University of Bath, BRE Centre for Innovative Construction Materials, Department of Architecture \\ and Civil Engineering, Claverton Down, Bath, UK
}

\begin{abstract}
Hygroscopic finishing materials can be used to moderate indoor humidity levels; they have the capacity to adsorb and release moisture from and to the surrounding air, depending on the indoor relative humidity levels. To determine the moisture buffering properties of materials several protocols have been introduced. However, testing procedures are based on a time-response method, where humidity variations are under a square wave function and temperature remains constant. Therefore, the ability of these methods to simulate material behaviour under real conditions, where cyclical humidity variations are more gradual, and temperature is variable, has been called into question. The aim of this study is to perform a standard moisture buffering test, by substituting the step-variation method, with a sinusoidal humidity function at different temperatures. Clay has been used to perform the tests in a climatic chamber, where a small increase of relative humidity have been set, in order to obtain a quasi-sinusoidal curve. The relative humidity variation are limited by low humidity $(33 \% \mathrm{RH})$ and high humidity $(75 \%$ $\mathrm{RH})$ and temperature variation between $18{ }^{\circ} \mathrm{C}$ and $28^{\circ} \mathrm{C}$. Materials tested present a lag in the response to the peak relative humidity to peak mass gain, which suggests an alternative way to consider the rate of sorption and the moisture storage function. The significance of the paper is to develop a laboratory test that can be more readily compared with the behaviour real buildings, which operate under more of a sine waveform
\end{abstract}

\section{Introduction}

Modern buildings have been made more air tight and highly insulated, to reduce heat losses. However, such buildings reduce the air and moisture exchange between the indoor and outdoor, influencing negatively occupant health and well-being. The lack of indoor air quality is not only related to the higher concentration of pollutant in the indoor, due to the reduction of ventilation, but also to the Relative Humidity (RH). Arundel et al. [1] explained that RH levels below $40 \%$ and above $60 \%$ influence thermal comfort and increase risks of exposure to bacteria, viruses and mould spore.

Mechanical devices, such as air conditioning systems, are commonly used to solve this problem and maintain optimal RH levels. However, such systems demand regular maintenance, good understanding of their operation and performance. In addition, there are concerns about noise production, costs and energy consumption [2].

\footnotetext{
* Corresponding author: v.cascione@bath.ac.uk
} 
In recent years designers and researchers have been looking for low energy design strategies, which bring into spotlight the moisture buffering concept in the indoor environment [3]. This property is the capacity of hygroscopic materials, like clay and gypsum, to adsorb and desorb water vapour from the indoor air, reducing the extreme highs and lows of RH in the indoor environment. The wider use of those materials on indoor surfaces has the ability to moderate indoor humidity fluctuations, potentially reducing operational energy use and improving indoor hygrothermal comfort and air quality.

Experimental tests, based on the step-response method such as the NORDTEST protocols [3], ISO 24353 [4] and JIS A 1470-1 [5], were developed to measure and compare the moisture buffering capacity of materials. These tests methods are applied in a controlled environment, in which temperature is constant and humidity varies, by following a square wave function [6]. The weight variation of samples is measured, when subjected to an adsorption phase for $8 \mathrm{~h}$, followed by a desorption step for $16 \mathrm{~h}$, varying cyclically the RH from $75 \%$ to $33 \%$.

The step-response tests do not represent the moisture buffering behaviour of finishing materials in real buildings, as it does not consider environmental factors, such as air change rate and daily outdoor temperature and RH variations. As Künzel et al. [7] showed, there is a strong inverse correlation between $\mathrm{RH}$ and temperature in both indoor and outdoor environment, which means that assuming constant temperature may not be adequate to understand the real behaviour of hygroscopic materials in the indoor. Therefore, daily RH variation are closer to a sinusoidal function, rather than a square wave function. It is consequently evident that the existing protocols are robust, repeatable, and simple, which make them suitable for materials performances comparison., However, the 'performance gap' between observations from real buildings and classification defined by NORDTEST point out to the necessity to develop a more complex laboratory sinewave test that should facilitate comparison with room/building testing.

This paper presents a new approach to measure moisture buffering in a laboratory scale testing, where RH follows a sinusoidal variation function and the effect of temperature on moisture buffering is considered. The aim of this study is to analyse the interaction between temperature and RH on moisture buffering and how temperature and humidity sinusoidal variation influence the dynamic sorption capacity of materials. The protocol is based on the NORDTEST set-up, where material mass variations are continuously recorded, to quantify the amount of water absorbed and desorbed during the humidity cycles. However, the change of the temperature and humidity profile leads to different material responses in the moisture adsorption. The significance of this research is to fill the gap between material testing and moisture buffering capacity of finishing material in real building, in order to help designers to quantify the moisture buffering impact in buildings.

\section{Materials and Method}

\subsection{Materials}

The main material analysed for this study was clay plaster. Only the undercoat was selected, as it has higher volume of involvement in moisture buffering, than the finishing layer [8]. The air dry clay plaster (Claytec base coat) was mixed with $20 \%$ mass of water by mechanical mixing in the laboratory. The mixing water amount was set according to the workability of the plaster. Specimens were cast in $150 \times 150 \times 20 \mathrm{~mm}$ moulds made with phenolic-faced plywood. Thereafter the specimens were stored for 28 days before testing in an environmental chamber at $20{ }^{\circ} \mathrm{C}$ and $60 \% \mathrm{RH}$. The dry density of clay, which is composed of $69 \%$ sand $(0-2 \mathrm{~mm}), 25 \%$ silt and $5 \%$ natural clay $(<5 \mathrm{~mm})$, is $1258 \mathrm{~kg} / \mathrm{m}^{3}$. 


\subsection{Methods}

Three specimens for each plaster were pre-conditioned, until the mass varied by less than $5 \%$. Specimens were placed on a mass scale inside the climatic chamber and covered by a screen to reduce the air speed to less than to $0.1 \mathrm{~m} / \mathrm{s}$. The mass of each specimen was measured every minute. Temperature and RH in the climatic chamber were monitored with humidity and temperature sensors. The new moisture buffering method used as foundation the NORDTEST protocol [3]. The specimens were exposed to six moisture buffering cycles. Each cycle consisted of 8 hours of high humidity and 16 hours of low humidity. Nevertheless, the RH profile was set to be sinusoidal instead of square wave. As the temperature in real buildings varies daily, temperature was also varied sinusoidally, in separate tests, inversely proportional to RH function.

To analyse the response of the plaster to temperature and $\mathrm{RH}$ sinusoidal variations singularly, two different types of tests were carried out: samples exposed to RH sinusoidal variation and constant temperature, and to variable temperature function and constant RH. The two test typologies were repeated three times respectively at different temperature $\left(18^{\circ} \mathrm{C}, 23^{\circ} \mathrm{C}\right.$ and $\left.28^{\circ} \mathrm{C}\right)$ and different $\mathrm{RH}(33 \%, 50 \%$ and $75 \%)$. The results of the test sets were then averaged, to obtain the response of the material to the combined effect of temperature and $\mathrm{RH}$ variations.

In Fig. 1 the experimental RH and temperature profile is shown. To define the high and low humidity interval, the moisture uptake is represented by the $61 \%$ to $75 \%$ curve and the moisture release phase by the $33 \%$ to $61 \%$ curve. For the temperature, the curve was set to start with 8 hours arch from $18.0^{\circ} \mathrm{C}$ to $21.3^{\circ} \mathrm{C}$, succeeded by a 16 hour ranging from $21.33^{\circ} \mathrm{C}$ to $28.0^{\circ} \mathrm{C}$. The temperature range was chosen, considering the acceptable operating temperature in a room based on ASHRAE Standard 55 [9, 10]. The Moisture Buffering Value (MBV) is expressed in $\mathrm{g} /\left(\mathrm{m}^{2} \% \mathrm{RH}\right)$.
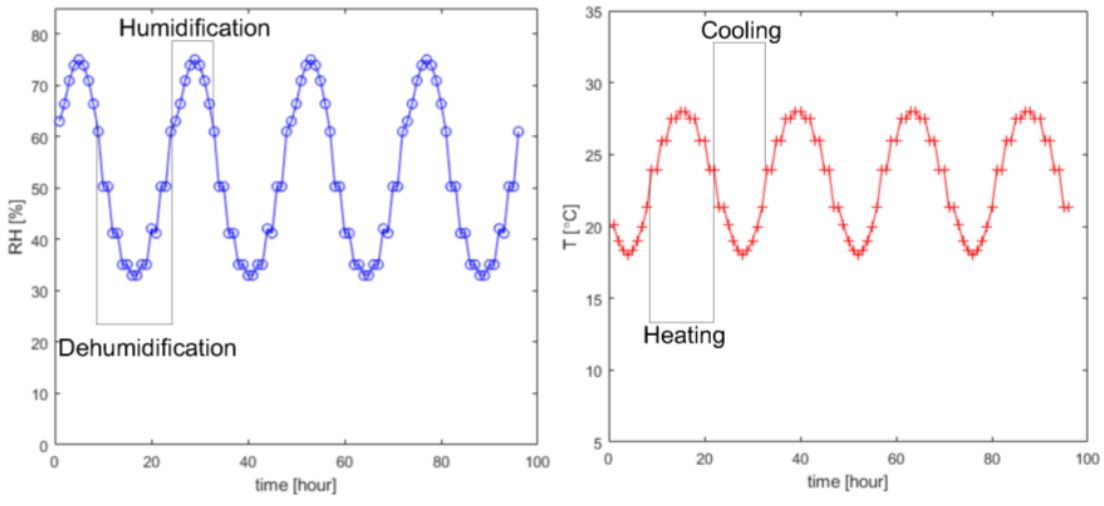

Fig. 1 RH and temperature variation profile

\section{Results}

The dynamic sorption curves are shown in Fig. 2. Fig. 2a illustrates the response of clay to the sinusoidal RH variation at three different temperatures. It is evident that the first cycle presents in all curves higher sorption values, as the specimens probably needed a lower preconditioning humidity. However, the sorption curve stabilises in the second cycle and presents a steady curve in all subsequent cycles. Observation of the single curves, the higher temperature yields a greater amplitude of sorption, while lower temperatures generates a smaller moisture uptake and release. At $28^{\circ} \mathrm{C}$ clay adsorbed $9.8 \mathrm{~g} / \mathrm{m}^{2}$ more than 
at $18^{\circ} \mathrm{C}$ and released $8.7 \mathrm{~g} / \mathrm{m}^{2}$ more (peak to average). However, independently to the temperature applied, all curves exhibited a synchronised delay of around 2 hours in response to $\mathrm{RH}$ variation peak points. Fig. $2 \mathrm{~b}$ represents the sorption response of clay to sinusoidal temperature fluctuation. Mass variation are significantly lower (between 6.8 and $9.8 \mathrm{~g} / \mathrm{m}^{2}$ peak to peak fluctuations). There is also not an appreciable difference between the curves (around 0.3-1.3 g/ $\mathrm{m}^{2}$ ), when subjected to different RH levels. However, the offset response of the material to temperature variations changes depending on the RH: all curves reach the peak before the temperature peak, but at $33 \%$ and $75 \% \mathrm{RH}$ the sorption peak is shifted of 1 hours and at $50 \%$ only 2 hour.

a)

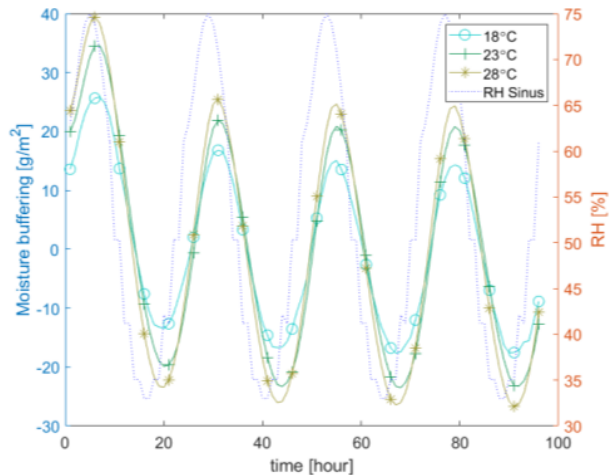

b)

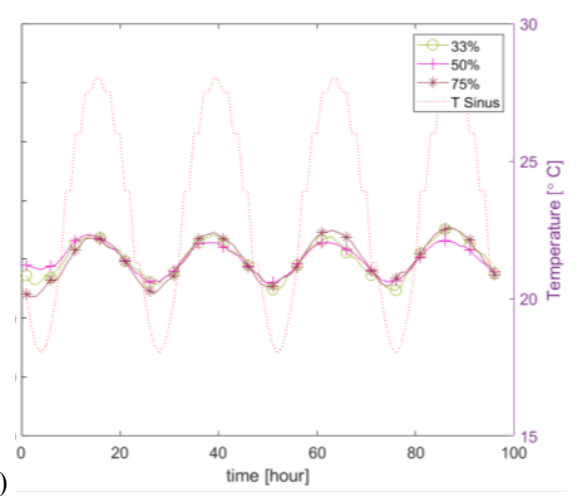

Fig. 2 Clay performance under RH variation at different temperature (a) and under temperature variation at different $\mathrm{RH}(\mathrm{b})$

\section{Analysis and discussion}

An example of the averaged curve with combined results from $\mathrm{RH}$ and temperature sinusoidal variation is shown in Fig. 3. The resultant curve was obtained numerically, by taking data from the tests at constant conditions of $23^{\circ} \mathrm{C}$ and $50 \% \mathrm{RH}$.

It is clear that the most influent factor on moisture buffering is the humidity fluctuation $\left(23.5 \mathrm{~g} / \mathrm{m}^{2}\right.$ peak to average), while temperature has a lower impact $\left(7.8 \mathrm{~g} / \mathrm{m}^{2}\right.$ minimum to average), but temperature is still influencing the moisture content in the plaster, as temperature sorption curves is opposite to the RH sorption one. For this reason, curves were averaged.

Fig. 4 shows the results of the combination of all curves. Dry and Humid corresponds to $33 \%$ and $75 \% \mathrm{RH}$ respectively, and Cold and Hot to $18^{\circ} \mathrm{C}$ and $28^{\circ} \mathrm{C}$. With this methods is possible to test the material performances in different climate, and evaluate where it works better.

Table 1 summarises the results obtained in Fig. 4. It is evident that the combination of $\mathrm{RH}$ and $\mathrm{T}$ variation reduces the sorption capacity, and in some cases shift the response time of the material. It is important to observe that, when temperature is low, the sorption capacity decreases, independently on the humidity level. It means that temperature plays an important role on moisture buffering and it cannot be excluded in the evaluation of the sorption capacity of materials. Moreover, clay responds better to dry environmental condition rather than humid, which indicates the plaster with a similar adsorption capacity, may have higher desorption rate and work better in drier areas. 


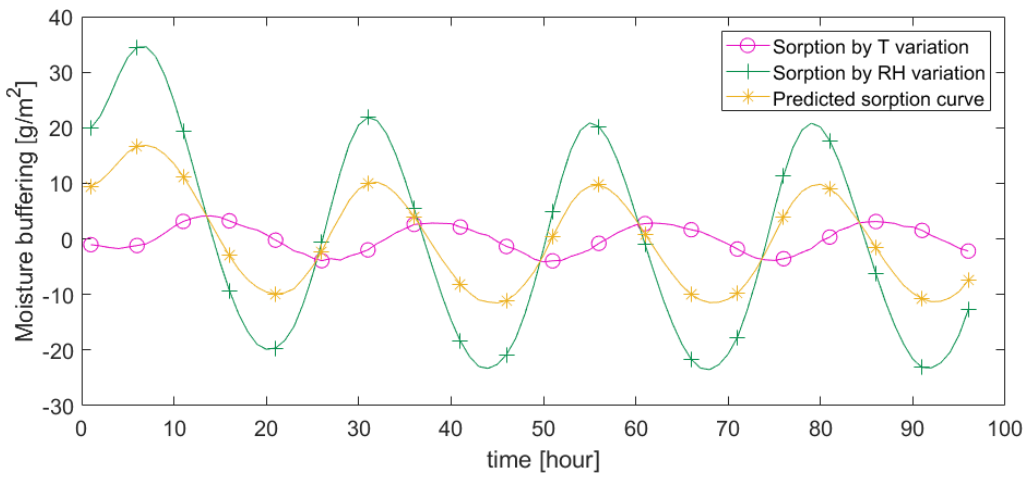

Fig. 3 Predicted curve with combined RH and temperature variation

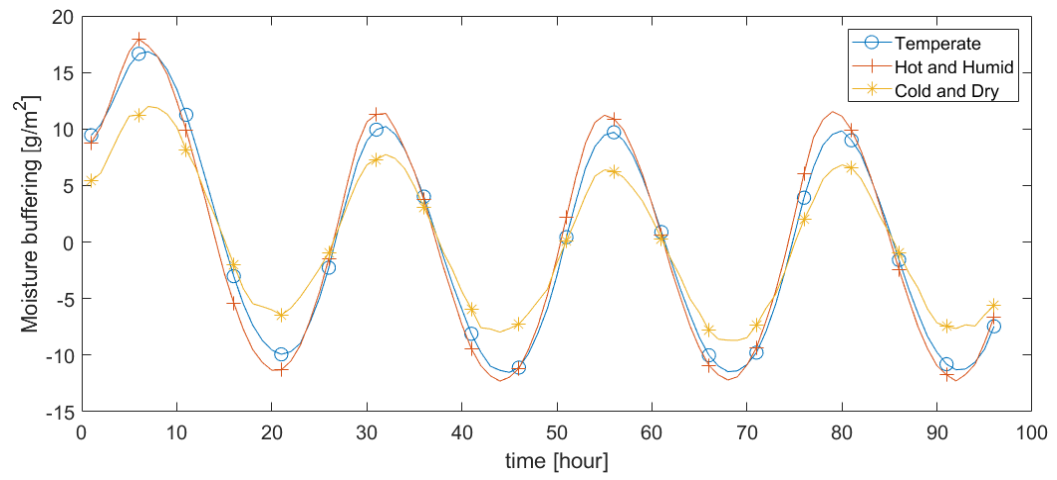

Fig. 4 Predicted curves for clay under combinations of different conditions

Table 1. Comparison and rating of resultant performance of clay under combinations of different conditions

\begin{tabular}{|c|c|c|c|c|}
\hline Curves & $\begin{array}{c}\text { Adsorption } \\
\left(\mathbf{g} / \mathbf{m}^{2}\right)\end{array}$ & $\begin{array}{c}\text { Desorption } \\
\left(\mathbf{g} / \mathbf{m}^{\mathbf{2}}\right)\end{array}$ & $\begin{array}{c}\text { Response offset } \\
\mathbf{( h )}\end{array}$ & $\begin{array}{c}\text { MBV } \\
\left(\mathbf{g} / \mathbf{~ m}^{\mathbf{2}} \mathbf{\%} \mathbf{R H}\right)\end{array}$ \\
\hline Cold and Humid & 7.72 & 6.84 & 2 & 0.34 \\
\hline Cold and Dry & 7.92 & 7.00 & 2 & 0.35 \\
\hline Mild & 11.24 & 9.97 & 2 & 0.50 \\
\hline Hot and Humid & 12.37 & 11.13 & 1 & 0.56 \\
\hline Hot and Dry & 12.64 & 11.28 & 1 & 0.57 \\
\hline
\end{tabular}

The analysis presented here gives only an indicative idea of the moisture buffering capacity in different climates, but it requires improvement and furtheer study. It is necessary to understand better the temperature fluctuation effect on materials, as clay never reaches a hygrothermal balance, when subjected to temperature variation. Therefore, in this study the results of the temperature and $\mathrm{RH}$ variation were averaged, while it may be necessary to define an influence ratio on moisture buffering of the two impact factors. Consequentely, materials should be tested with simultaneus $\mathrm{RH}$ and temperature sinusoidal variation.

Table 1 shows the MBV values obtained from this test, which are below the moderate class in the NORDTEST classification, and they are significantly lower than the equivalent 
values obtained in the standard NORDTEST. It means that this range should be rearranged, and more materials need to be tested.

\section{Conclusion}

Moisture buffering capacity of clay was determined experimentally, by substituting the humidity square curve variation in the NORDTEST with a sinusoidal curve. The modified test considers indoor $\mathrm{RH}$ as a harmonic function, and introduces also the influence of temperature fluctuation on moisture buffering. As the impact of temperature on the clay sorption process was not known, two different tests were performed to understand individually the effect of temperature and $\mathrm{RH}$ sinusoidal variation on the specimens. The obtained results were then combined, to represent the behaviour of the material in different climatic zones.

The study presented here attempted to analyse the impact of simultaneous RH and temperature variation on hygroscopic materials, but further analysis are necessary. However, this method improves the existing protocols, as it does not only compares the moisture buffering performances of materials, but it also gives information on the sorption capacity of a finishing material in different climatic zones and their response to sinusoidal humidity variation.

The significance of this new method is to improve moisture buffering testing, optimising material use to increase indoor environment quality. An analysis of material performances in different climatic zones, and under sinusoidal temperature and $\mathrm{RH}$ variations, can lead to have a more realistic understanding of the impact of finishing materials on the hygrothermal comfort and to the consequent reduction of "active" conditioning system energy consumption.

This study was supported by the EPSRC Centre for Decarbonisation of the Built Environment (dCarb) [grant number EP/L016869/1] and a Building Research Establishment (BRE) Scholarship.

\section{References}

1. A. Arundel, E. M. Sterling, J. H. Biggin, and T. D. Sterling., Environmental Health Perspectives, 65,351 (1986)

2. D. Crump, A. Dengel, and M. Swainson, NHBC Foundation (2009)

3. C. Rode, R. Peuhkuri, L.H. Mortensen, K. Hansens , B. Time, A. Gustavsen, T. Ojanen, J, Ahonen, K, Svennberg, L.E. Harderup, J. Arfvidsson J, Technical University of Denmark (2005)

4. ISO $24353(2008)$

5. JIS 1470-1 (2002)

6. V. Cascione, D.Maskell, A.Shea, P.Walker, Construction and Building Materials. 200, 333-343 (2019)

7. H.M. Künzel, D. Zirkelbach, and K. Sedlbauer, Proceedings of 1st International Conference on Sustainable Energy and Green Architecture (2003)

8. D. Maskell, A. Thomson, P. Walker, and M. Lemke, Building and Environment 130,143-50 (2018)

9. ASHRAE Standard 55 (2013)

10. G.D. Kontes, G. I. Giannakis, P. Horn, S. Steiger, and D. V Rovas, Energies, 10, 9,1368 (2017) 